RESEARCH ARTICLE

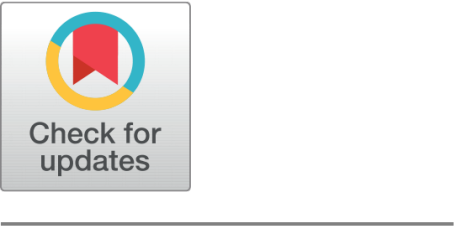

G OPEN ACCESS

Received: 06.11.2021

Accepted: 04.12.2021

Published: 28.12 .2021

Citation: Manthesha, Mallikarjun PB, Kavitha L, Shobha KC (2021) Mixed Convection of Williamson Fluid along an Inclined Porous Microchannel with Chemical Reaction by taking Non-Constant Thermal Conductivity: An Entropy Analysis . Indian Journal of Science and Technology 14(48): 3525-3536. https://doi.org/

10.17485/IJST/v14i48.1853

* Corresponding author.

mbp1007@yahoo.com

Funding: None

Competing Interests: None

Copyright: (c) 2021 Manthesha et al. This is an open access article distributed under the terms of the Creative Commons Attribution License, which permits unrestricted use, distribution, and reproduction in any medium, provided the original author and source are credited.

Published By Indian Society for Education and Environment (iSee)

ISSN

Print: 0974-6846

Electronic: 0974-5645

\section{Mixed Convection of Williamson Fluid along an Inclined Porous Microchannel with Chemical Reaction by taking Non-Constant Thermal Conductivity: An Entropy Analysis}

\author{
Manthesha ${ }^{1}$, Patil B Mallikarjun ${ }^{2 *}$, L Kavitha ${ }^{3}$, K C Shobha ${ }^{1}$ \\ 1 Research Scholar, Department of Studies and Research in Mathematics, Tumkur University, \\ Tumakuru, 572103, Karnataka, India \\ 2 Professor, Department of Studies and Research in Mathematics, Department of \\ Mathematics, Tumkur University, Tumakuru, 572103, Karnataka, India \\ 3 Assistant Professor, Department of Mathematics, REVA University, Bengaluru, 560064 , \\ Karnataka, India
}

\section{Abstract}

Objective: Mixed convection of Williamson fluid along an inclined porous microchannel with the influence of first-order chemical reaction is considered. The thermal conductivity kept varying throughout the flow. Boundaries of the channel are maintained with slip and jump conditions. With these conditions in this present article we are aimed to analyse the velocity, heat transfer and entropy generation in the flow. Method: The non-linear equations which govern the flow are tackled utilizing the bvp4c technique which involves the finite difference method and improved by using the Lobatto III formula. Findings: Fluid flow, heat transfer and entropy production analysis are done for the various estimations of the parameters which are affecting the flow, and the study highlights that non constant thermal conductivity and mixed convection parameters have to be maintained at lower values for the efficient energy transfer in the model. Entropy production shows the dual trend for distinct estimations of Weinsenberg number. Applications: The obtained result in the present study helps industries to analyse the efficient energy transfer in their engineering designs and thermal equipment's. Also the flow of nonNewtonian fluid has applications in the field of blood flow, lubrication and in many engineering devices such as micro heat exchangers, micro mixers, micro cooling systems.

Keywords: Williamson fluid; Inclined microchannel; Variable thermal conductivity; Entropy production; Bejan number

\section{Introduction}

The flow in microchannel is an active area of study in the recent days due to its significance in the field of science and engineering. Fluid movement in microchannel 
involves the high rate of heat transfer due to its volume and surface ratio. Many of the mechanical and engineering equipment liberate heat at the time of their operations this leads to the loss in energy. To minimize the energy loss many micro cooling systems are introduced. Due to boundless applications the flow in microchannel has attracted many researchers and scientists to study in this area.

Makinde and Eegunjobi ${ }^{(1)}$ looked into the movement of Casson fluid along a microchannel and analysed the irreversibility of the system. The flow of Williamson fluid flow along a plate which is moving is taken for study by Zaib et al. ${ }^{(2)}$, the results of the study highlights that movement of the fluid is suppressed by Williamson parameter. A theoretical investigation is conducted to analyse the movement of Williamson liquid along a stretching sheet by Khan et al. ${ }^{(3)}$, Kumar et al. ${ }^{(4)}$. Nanofluid flow along a microchannel and the entropy production from it is studied and analysed by Gireesha et al. ${ }^{(5)}$ and Saima et al. ${ }^{(6)}$. Sahoo and Nandkeoiyar ${ }^{(7)}$ reported a study of both forced and free convection of casson nanofluid with the effect of thermal radiation. Salahuddin and Malik ${ }^{(8)}$ used Keller box method effectively to study the Williamson fluid flow through a cylinder. Jha and Aina ${ }^{(9)}$ contribute their work to investigate the mixed convection through a microchannel by sucking and injecting the fluid. The work done by Hang and Qiang ${ }^{(10)}$, Patil et al. ${ }^{(1)}$ and Shobha et al. ${ }^{(12)}$ are the good literature for the study of mixed convection through channels. Ibrahim and Anbessa ${ }^{(13)}$ looked into the Williamson nano fluid flow along stretching sheet and results shows that enhancement in Williamson parameter leads to decelerate the fluid temperature. An analysis is made by Cimpean and Pop ${ }^{(14)}$ to investigate the both free and forced flow of nano fluid along a porous filled channel, for the study they considered three nano fluid namely Al2O3-H2O,TiO2-H2O.Cu-H2O. Srinivasacharya and Bindu ${ }^{(15)}$ looked into the micropolar fluid flow along an inclined channel and the flow problem is tackled using spectral quasi linearization technique results attained shows that angle of inclination and the Brinkman number should be maintained at lower values to minimize the entropy. Zaidi and Ahmad ${ }^{(16)}$ made an investigation to analyse the flow along an inclined channel the channel is equipped with the slip and jump boundary, the attained results effectively discussed using the graphs.

A theoretical analysis of the Casson fluid flow along an inclined micro-annular channel is done by Idowu et al. ${ }^{(17)}$. Gireesha et al. ${ }^{(18)}$ constructed a model which includes both inclined and horizontal convection of Williamson nano fluid and analysed the flow. Mohd et al. ${ }^{(19)}$ considered the flow of ferro fluid along a boundary layer and reported that Fe3O4- $\mathrm{H} 2 \mathrm{O}$ has lower heat transfer rate than the Fe3O4- kerosene ferrofluid. The contribution done by the authors Venkateswarlu et al. ${ }^{(20)}$, Patil et al. $^{(21)}$, Xiaobio and Xuegong ${ }^{(2)}$ are the good results for the study of fluid flow through vertical channel with the influence of chemical reaction. Abbas et al. ${ }^{(23)}$ considered the Jeffrey fluid flow in a porous channel and analysed the impact of chemical reaction on it. Ziagta ${ }^{(24)}$ contributed their work to analyse the both forced and free convection along a vertically placed channel by considering the chemical reaction effect. Umavathi et al. ${ }^{(25)}$ looked into the study of free flow along a rectangular duct by maintaining non constant thermal conductivity.

Alireza ${ }^{(26)}$ reported good results for the study of entropy generation. Makinde and Eegunjobi ${ }^{(27)}$ reported the influence of convictive heating on the fluid flow. Anurag et al. ${ }^{(28)}$ looked into the Casson fluid flow along microchannel. Krishna ${ }^{(29)}$ and Renukadeve et al. ${ }^{(30)}$ devoted their study to analyse the entropy generation in the flow with the influence of heat sink. Zahra et al. ${ }^{(31)}$ contributed their work to study the bioconvection of Williamson nanofluid along a stretching sheet, and found that slip parameters influences the velocity, temperature of the flow. Ellahi et al. ${ }^{(32,33)}$ looked into the entropy generation analysis and obtained interesting results for the boundary layer flow over a moving plate and flow of nanofluid through porous medium. An investigation is conducted by Shaheen et al. ${ }^{(34)}$ to study the flow of hyperbolic nanofluid in a ciliated tube they found that addition of nano particles increases the thermal conductivity of the fluid. Bhatti et al ${ }^{(35)}$ examined the convection of Williamson hydromagnetic nanofluid and reported the important results on the entropy generation in the flow.

We found good literature in the study of Williamson fluid flow along different channels with the influence of various parameters. Recently Gireesha et al. ${ }^{(2)}$ studied Williamson fluid flow along the horizontal and inclined channels. They acknowledge that Weissenberg, Reynolds number amplifies the heat transfer rate.

But so far, there is no study is found to analyse the entropy generation in the mixed convection of Williamson fluid along the micro porous channel with the influence of chemical reaction, variable thermal conductivity. So in order to fill this research gap in this present article we aimed to analyse the entropy generation for the flow of Williamson fluid along the inclined micro porous channel with the influence of chemical reaction which includes activation energy in it and by taking thermal conductivity as a variable property.

\section{Mathematical flow formulation}

Convection of Williamson fluid is along an inclined microchannel with the inclination ' $\alpha$ ' is taken for the study and the two plates of the microchannel is separated by distance 'a' and the temperature of the plates are at distinct temperatures $T_{h}$ and $T_{a}$. The physical configuration of the considered flow model is displayed in figure 1 .

Following are the assumptions we adopted for our present study, 
- The flow is taken as viscous, laminar and steady.

- The thermal conductivity of the fluid taken as variable property whereas the density and viscosity of the fluid are taken as constant.

- The flow is carried out through the porous medium with the influence of magnetic field.

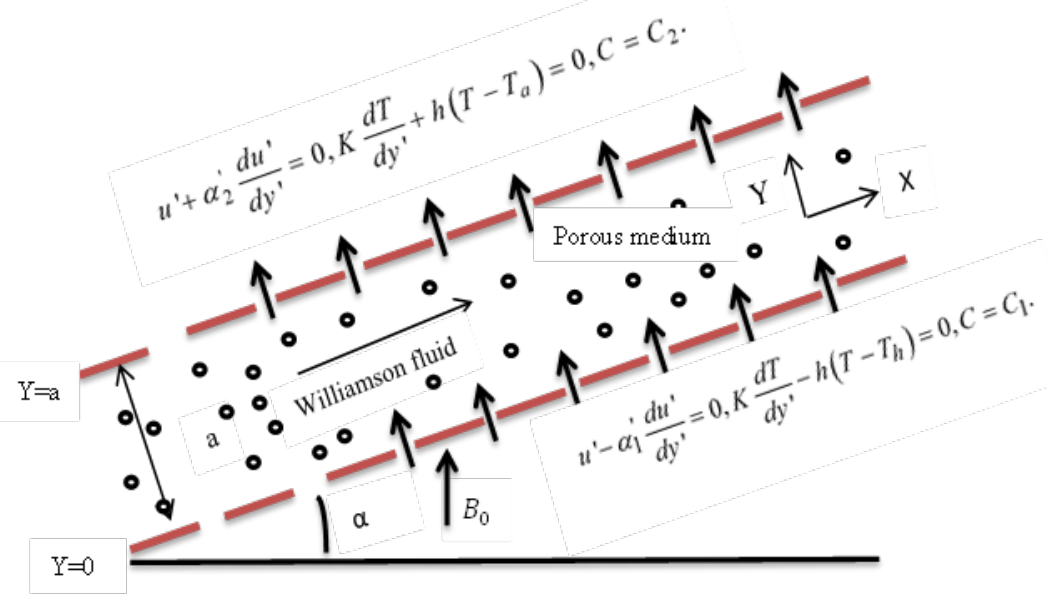

Fig 1. Flow configuration

Under the above mentioned conditions the momentum, temperature distribution and chemical reaction equations in dimensional form takes the form as below ${ }^{(5,18)}$ :

$$
\begin{gathered}
-\frac{d p}{d x}+\mu \frac{d^{2} u^{\prime}}{d y^{\prime 2}}+\sqrt{2} \mu \Gamma \frac{d u^{\prime}}{d y^{\prime}} \frac{d^{2} u^{\prime}}{d y^{\prime 2}}+\rho g \beta\left(T-T_{a}\right) \sin \alpha \\
\quad+\rho g \beta\left(C-C_{0}\right)-\sigma B_{0}^{2} u^{\prime}-\frac{\mu}{k} u^{\prime}=0 \\
K\left(\frac{d^{2} T}{d y^{\prime 2}}\right)+\mu\left(\frac{d u^{\prime}}{d y^{\prime}}\right)^{2}+\frac{\mu}{k} u^{\prime 2}+\sigma B_{0}^{2} u^{\prime 2} \pm Q\left(T-T_{a}\right)=0 \\
D\left(\frac{d^{2} C}{d y^{\prime 2}}\right)-\gamma\left(C-C_{0}\right)=0
\end{gathered}
$$

Employed boundary conditions for the considered flow model in dimensional form,

$$
\begin{gathered}
u^{\prime}-\alpha_{1}^{\prime} \frac{d u^{\prime}}{d y^{\prime}}=\left.0\right|_{y=0}, u^{\prime}+\alpha_{2}^{\prime} \frac{d u^{\prime}}{d y^{\prime}}=\left.0\right|_{y=a} \\
K \frac{d T}{d y^{\prime}}-h\left(T-T_{h}\right)=\left.0\right|_{y^{\prime}=0}, K \frac{d T}{d y^{\prime}}+h\left(T-T_{a}\right)=\left.0\right|_{y^{\prime}=a}, \\
C=\left.C_{1}\right|_{y^{\prime}=0}, C=\left.C_{2}\right|_{y^{\prime}=a}
\end{gathered}
$$

Thermal conductivity of the Williamson fluid is dependent on temperature and varied throughout the flow is denoted as:

$$
K=k(1+\varepsilon \theta)
$$

Where $\varepsilon$ - variable thermal conductivity parameter.

Dimensionless variables used for the non-dimensionalization

$$
y=\frac{y^{\prime}}{a}, \quad \theta=\frac{\left(T-T_{a}\right)}{\left(T_{h}-T_{a}\right)}, \quad u=\frac{u^{\prime}}{u_{0}}
$$


Dimensionless form of the governing equations $(1,2)$ using $(6)$ are

$$
\begin{gathered}
\left(1+W e \frac{d u}{d y}\right) \frac{d^{2} u}{d y^{2}}+\lambda \sin \alpha \theta+\lambda_{C} \phi-M^{2} u-\sigma^{2} u+P=0 \\
(1+\varepsilon \theta) \frac{d^{2} \theta}{d y^{2}}+B r\left(\frac{d u}{d y}\right)^{2}+B r M^{2} u^{2}+B r \sigma^{2} u^{2} \pm \psi \theta=0 \\
\frac{d^{2} \phi}{d y^{2}}-\xi^{2} \phi=0
\end{gathered}
$$

Boundary conditions in dimensionless form,

$$
\begin{gathered}
u-\delta \frac{d u}{d y}=\left.0\right|_{y=0}, u+\delta \frac{d u}{d y}=\left.0\right|_{y=1} \\
\frac{d \theta}{d y}-B i(\theta-1)=\left.0\right|_{y=0}, \frac{d \theta}{d y}+B i \theta=\left.0\right|_{y=1} \\
\phi=-\left.1\right|_{y=0}, \phi=\left.1\right|_{y=1} .
\end{gathered}
$$

where $\mathrm{Re}=\frac{\rho a u_{0}}{\mu_{0}}$ Reynolds number, $\mathrm{Gr}=\frac{g \beta\left(T_{h-}-T_{a}\right) a^{3} \rho^{2}}{\mu_{0}^{2}}, \mathrm{Bi}=\frac{a h}{k}$ Biot number, $\theta_{h}=\frac{T_{h}}{T_{a}}$ characteristic temperature ratio, $\mathrm{Br}=E_{c} \mathrm{Pr}$

- Brinkman number, $G r_{c}=\frac{g \beta\left(C_{1}-C_{0}\right) a^{3} \rho^{2}}{\mu_{0}^{2}}, \varepsilon=$ variable thermal conductivity parameter. $\lambda=\frac{G r}{R e}$ mixed convection parameter, $\sigma^{2}=\frac{a^{2}}{K}$ porous parameter, $\lambda_{C}=\frac{G r_{c}}{R e}$ concentration buoyancy parameter. $\xi=$ concentration parameter. $\delta=$ slip parameter.

\subsection{Analysis of thermodynamic second law}

Entropy is the evaluation of the irreversibility in the model. To obtain the efficient flow model it necessary to minimize the entropy production in the model. Entropy analysis can be done second law of thermodynamics. In present considered flow model magnetic field, permeability, viscous dissipation and heat transfer are affecting the entropy production in the flow.

The equation of irreversibility takes the form as:

$$
E_{g}=\frac{1}{T_{a}^{2}} K\left(\frac{d T}{d y^{\prime}}\right)^{2}+\frac{\mu}{T_{a}}\left(\frac{d u^{\prime}}{d y^{\prime}}\right)^{2}+\frac{\mu}{T_{a} K} u^{\prime 2}+\frac{1}{T_{a}} \sigma B_{0}^{2} u^{\prime 2}
$$

On non-dimensionalising, equation of irreversibility of the system reduces to:

$$
N s=\frac{E_{g}}{E_{g_{0}}}=(1+\varepsilon \theta)\left(\frac{d \theta}{d y}\right)^{2}+\frac{B r}{T_{P}}\left(\frac{d u}{d y}\right)^{2}+\frac{B r}{T_{P}} M^{2} u^{2}+\frac{B r}{T_{P}} \sigma^{2} u^{2}
$$

where $E_{g_{0}}=\frac{k}{T_{a}^{2}} \frac{\Delta T^{2}}{a^{2}}-$ characteristic entropy generation.

Above equation can be written as,

$$
N s=N_{h}+N_{v}+N_{P}
$$

$N_{h}=$ Entropy due to transfer of heat, $N_{v}=$ Entropy due to viscous dissipation, $N_{P}=$ Entropy due to porous medium.

The ratio of entropy production due to heat transfer to the overall entropy production is denoted as Bejan number, it is defined as:

$$
B e=\frac{N_{h}}{N_{h}+N_{v}+N_{P}}
$$




\section{Numerical procedure}

The reduced system of nonlinear equations (9), (10) and (11) and the corresponding equations of the employed boundary conditions for the considered flow are (12), (13) and (14) to solve these equations we used the Bvp4c Technique which involves the finite difference method. By this, we transform the ODE, by assigning new variables.

Assuming, $u=y_{1}, u^{\prime}=y_{2}, \quad \theta=y_{3}, \theta^{\prime}=y_{4}$,

Transforming the equations (9), (10) and (11) using the above assumptions we can write,

$\left[\begin{array}{l}y_{1}^{\prime} \\ y_{2}^{\prime} \\ y_{3}^{\prime} \\ y_{4}^{\prime} \\ y_{5}^{\prime} \\ y_{6}^{\prime}\end{array}\right]=\left[\begin{array}{l}y_{2} \\ \sigma^{2} y_{1}+M^{2} y_{1}-\lambda \sin \alpha-\lambda_{C} y_{5}-P /(1+\text { Wey }) \\ y_{4} \\ \frac{-B r y_{2}^{2}-B r \sigma^{2} y_{1}^{2}-B r M^{2} y_{1}^{2}-\psi y_{3}}{1+\varepsilon y_{3}} \\ y_{6} \\ \xi^{2} \phi\end{array}\right]$

and the boundary conditions can be written as,

$y_{1}(0)=\delta y_{2}, y_{1}(a)=-\delta y_{2}, y_{4}(0)=B i(\theta-1), y_{4}(a)=-B i \theta, y_{6}(0)=-1, y_{4}(a)=1$.

\section{Results and discussion}

The considered flow model is solved numerically and the solutions for velocity, temperature, entropy generation and Bejan number are obtained and the influence of chemical reaction parameter, variable thermal conductivity parameter, Biot number, mixed convection parameter, concentration buoyancy parameter, and inclination are discussed using the graphs. The results calculated by maintaining constant values as: $\mathrm{P}=1, \mathrm{Br}=0.7, \lambda=2, \mathrm{Bi}=1, \mathrm{We}=0.5, \mathrm{M}=1, \alpha=\pi / 4, \sigma=0.5, \phi=1, \varepsilon=0.1, \lambda_{C}=2, \xi=0.5$, $\delta=0.1$.

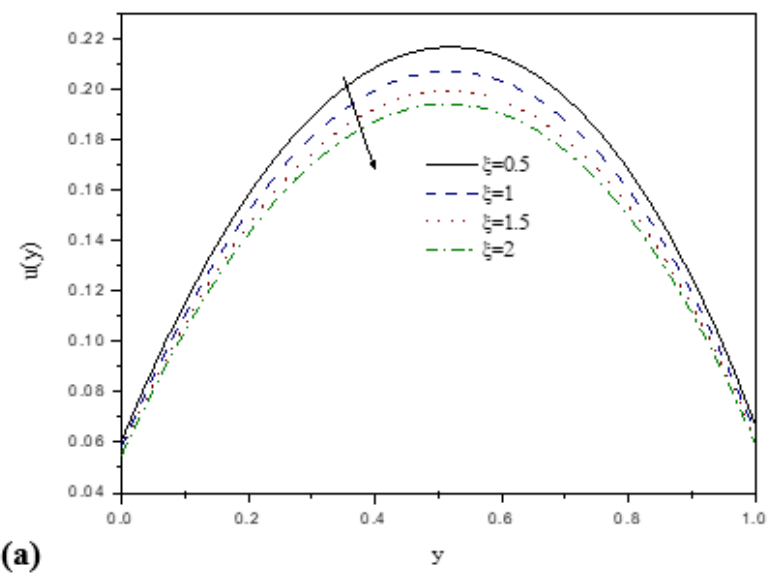

(a)

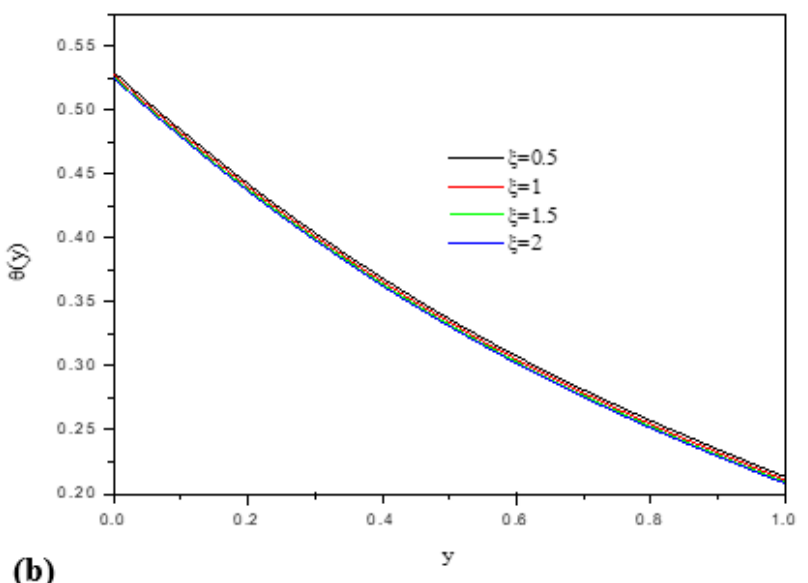

(b)

Fig 2. Impact of $\xi$ on the velocity (a) and temperature (b)

Figure $2 \mathrm{a}$ is the velocity profile for the variation of chemical reaction parameter. With the increase in the concentration the movement of the fluid particles is reduced due to this we observe in the figure that with the accelerated values of $\xi$ velocity of the fluid decelerates. In Figure $2 b$ with magnification in chemical reaction parameter due to absorption of heat the temperature profile supresses. Variation of velocity and temperature with the influence of $\mathrm{Br}$ is displayed Figure 3a and Figure $3 \mathrm{~b}$. As the Brinkman number increases that leads to the viscous dissipation hence here it is noted that Brinkman number makes significant effect on the flow and hence here we observe that with the improved estimations of Brinkman number the temperature and velocity profile shows the enhanced nature.

Figure $4 \mathrm{a}$ to Figure $4 \mathrm{c}$ present the temperature profile, entropy production and Bejan number for the different values of $\varepsilon$. It is interestingly observed in Figure 4a that with the enhanced values of the $\varepsilon$ the temperature profile accelerates and with the higher values of non-constant thermal conductivity parameter entropy production rises in the system it can be seen in Figure 4 b. Entropy generation due to heat transfer is more compared to the overall entropy generation in the system hence 


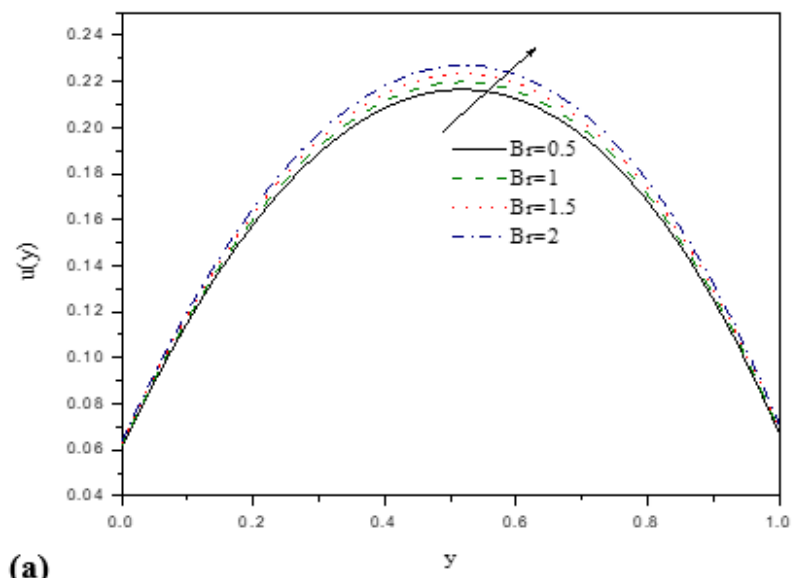

(a) y

Fig 3. Impact of $\mathrm{Br}$ on the velocity (a) and temperature (b)

(b)

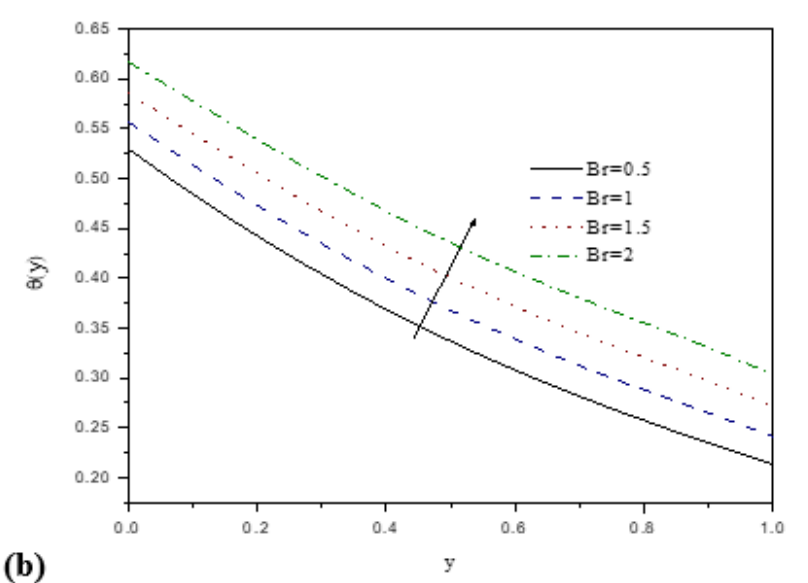

y 
(a)
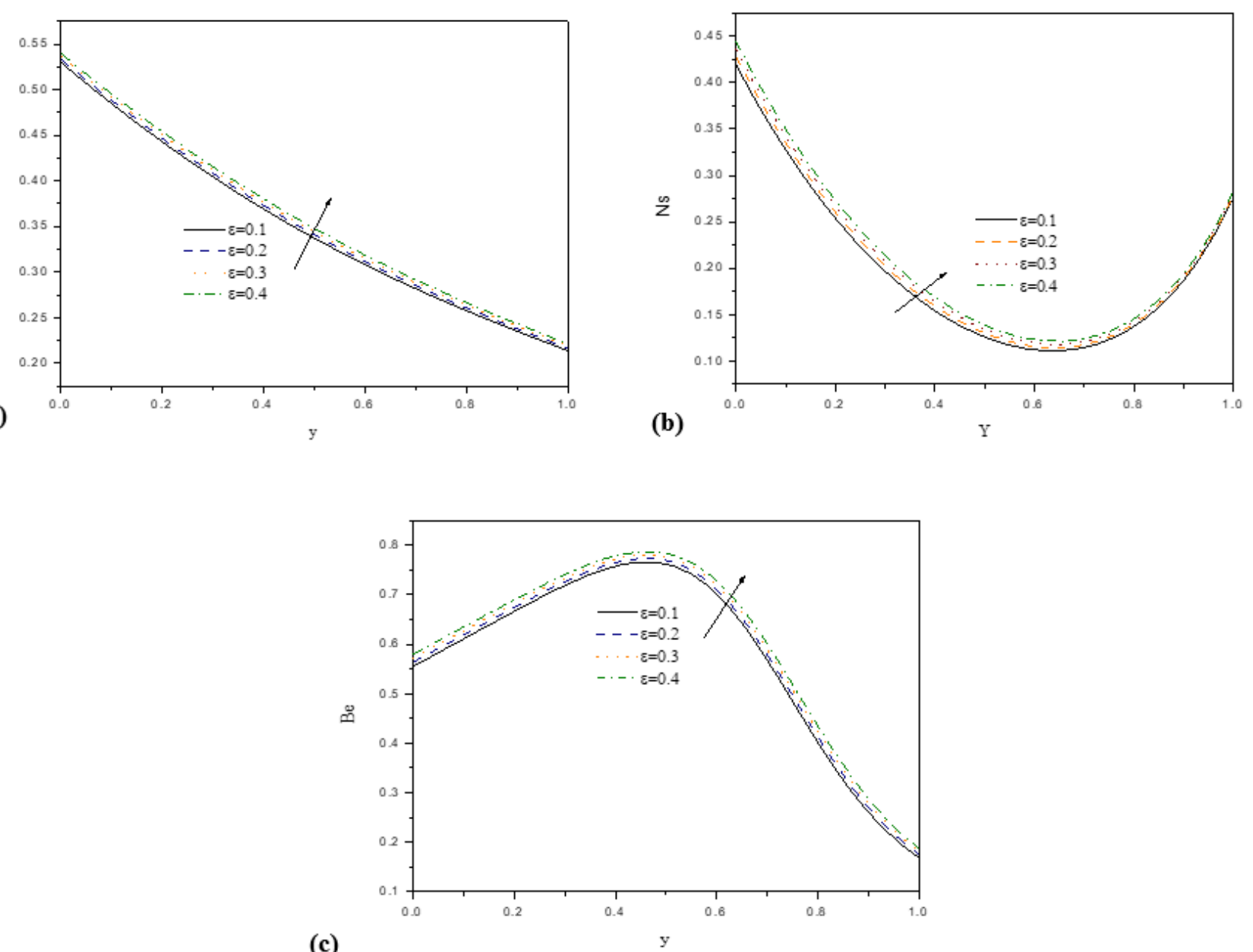

(c)

Fig 4. Impact of $\varepsilon$ on the temperature (a), entropy generation (b) and Bejan number (c)
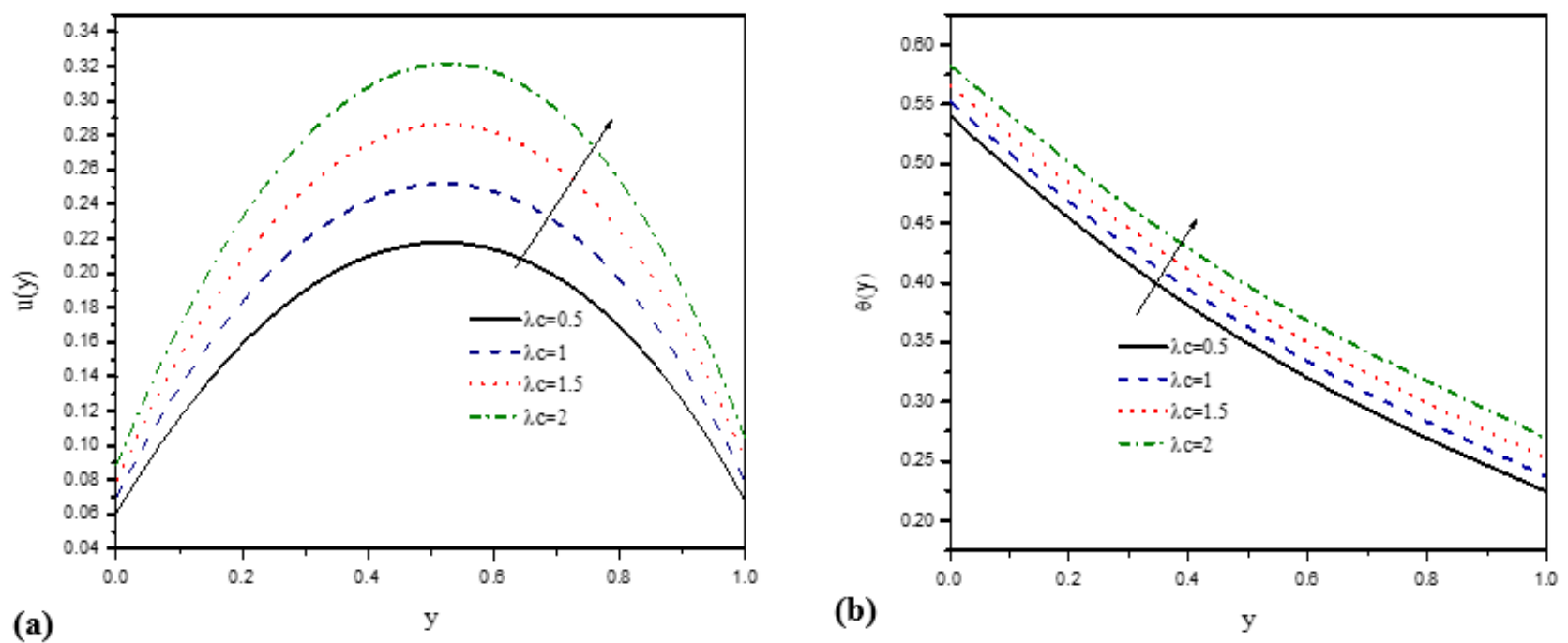

Fig 5. Impact of $\lambda_{C}$ on the velocity (a) and temperature (b) 

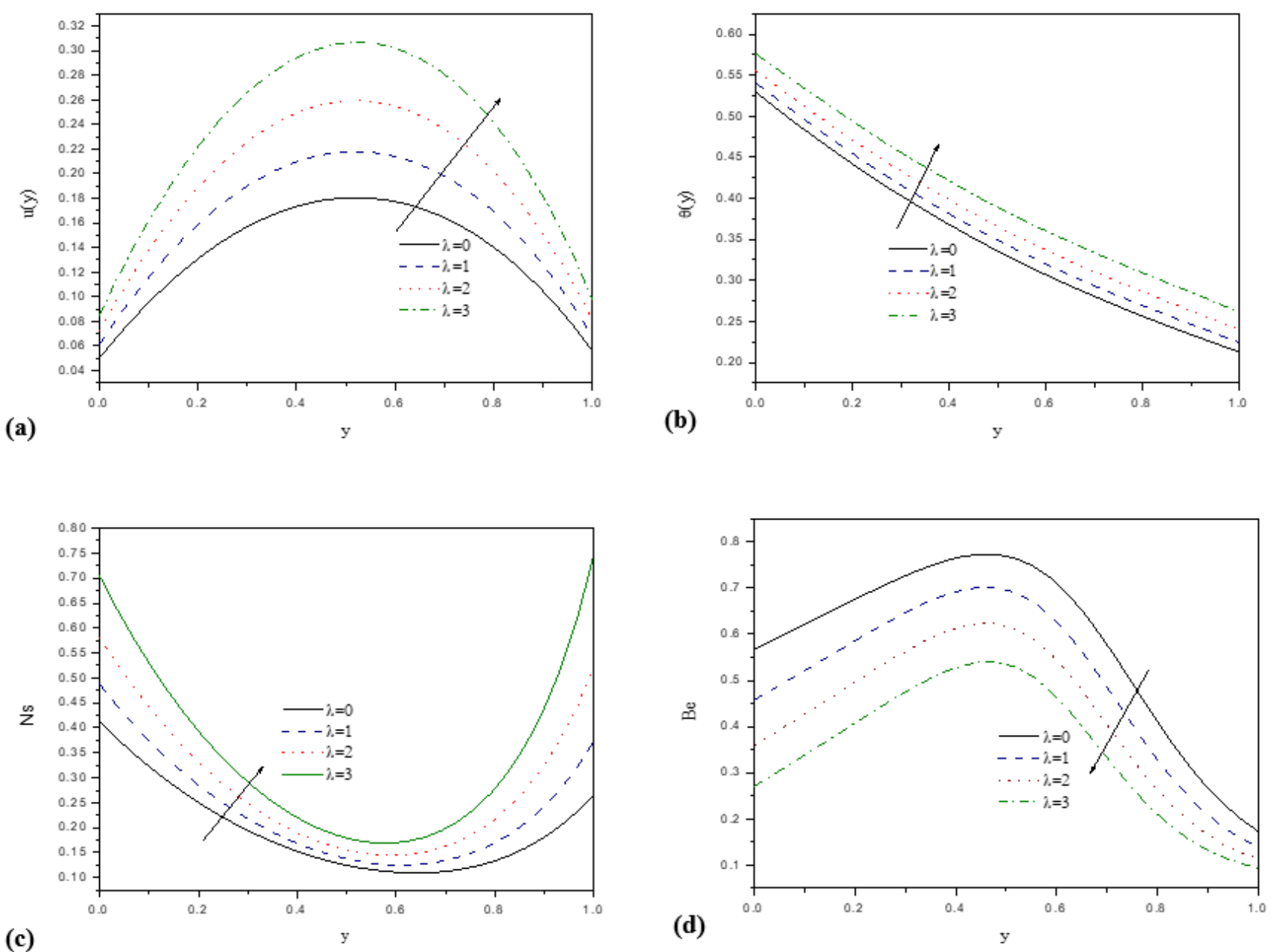

Fig 6. Impact of $\lambda$ on the velocity (a), temperature (b), entropy generation (c) and Bejan number (d).

Table 1. The comparison ofnumerical values of velocity with the published work of Gireesha et al. ${ }^{(18)}$ and Makinde and Eegunjobi ${ }^{(27)}$

\begin{tabular}{llll}
\hline $\mathrm{y}$ & Makinde and Eegunjobi ${ }^{(27)}$ & Gireesha et al. $^{(18)}$ & Present study \\
\hline 0 & 0.0000000 & 0.0000000 & 0.000000 \\
0.2 & 0.0711487 & 0.0711487 & 0.071149 \\
0.4 & 0.0963903 & 0.1137694 & 0.113770 \\
0.6 & 0.1215460 & 0.1215460 & 0.121550 \\
0.8 & 0.0867637 & 0.0867637 & 0.086764 \\
1 & 0.0000000 & 0.0000000 & 0.000000 \\
\hline
\end{tabular}

the display of the numerical solution obtained for the velocity for the limiting case. The obtained results are in good agreement with theexisting studies Gireesha et al. ${ }^{(18)}$ and Makinde and Eegunjobi ${ }^{(27)}$. The result is obtained by maintaining $\mathrm{We}=\lambda_{C}=\lambda=\mathrm{M}=\sigma=\psi=0 . \mathrm{P}=1, \mathrm{Br}=1$. 

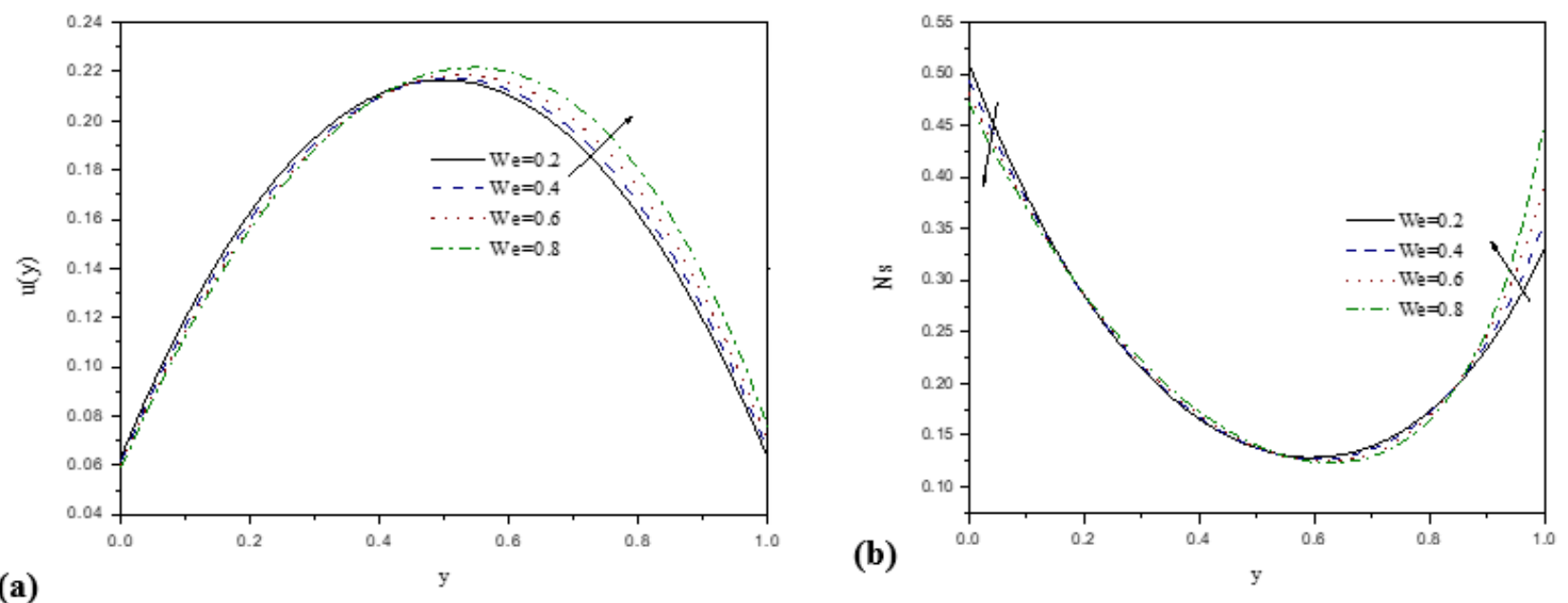

(a)

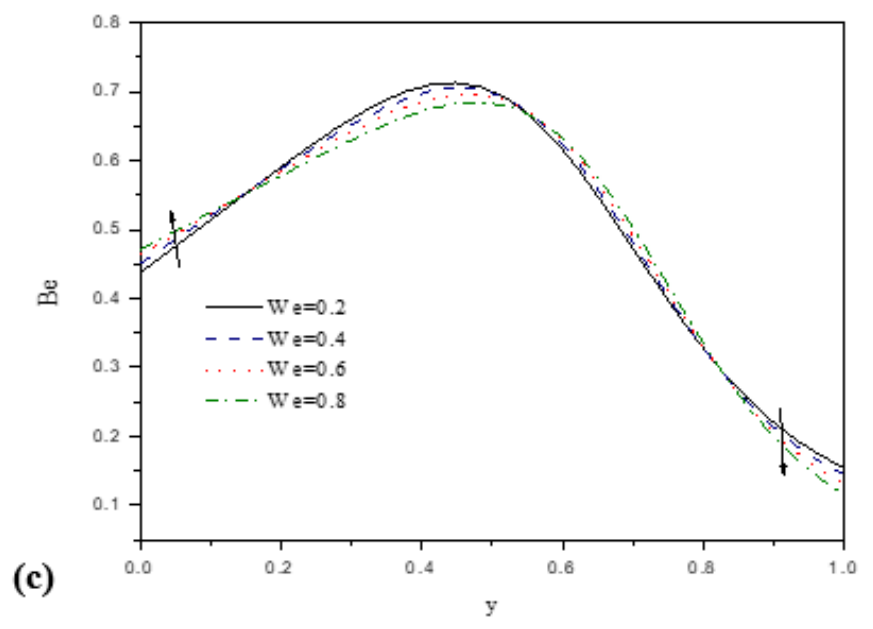

Fig 7. Impact of We on the velocity (a), entropy generation (b) and Bejan number (c)

\section{Conclusion}

The entropy generated in the mixed convection of Williamson fluid along an inclined porous microchannel with the influence of variable thermal conductivity and chemical reaction in the presence of magnetic field is studied. The major outcomes of the present study are, porous

- $\xi$ reduces the temperature and velocity of the fluid.

- Concentration buoyancy parameter, $\mathrm{Br}, \varepsilon$, We, $\mathrm{Bi}$ and $\lambda$ improves the velocity and temperature of the fluid.

- Magnified values of $\varepsilon, \lambda, B$ and inclination of the channel enhance the entropy production.

- Increase in We shows the dual trend for the entropy production and Bejan number.

- Due to the dominant of the entropy production of the system to the entropy production due to heat transfer, Bejan number shows the declining trend for the $\lambda$. channel inclination.

- Bejan number shows upward nature for Bi and variable thermal conductivity parameter. 


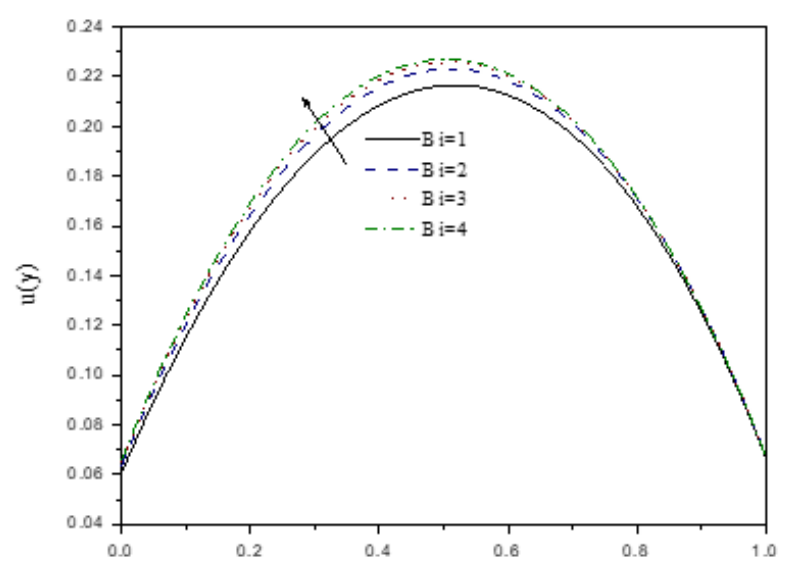

(a)

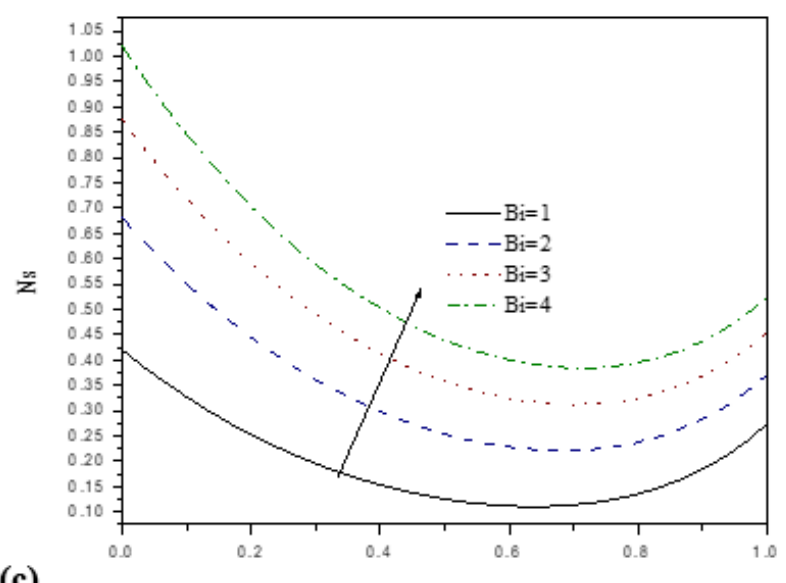

(c) y



(b)

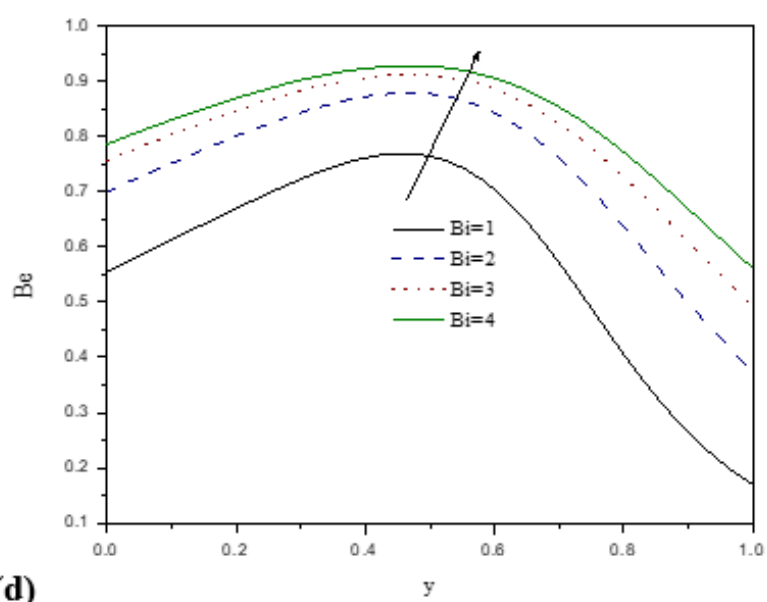

Fig 8. Impact of $\mathrm{Bi}$ on the on the velocity (a), temperature (a), entropy generation (b) and Bejan number (c)

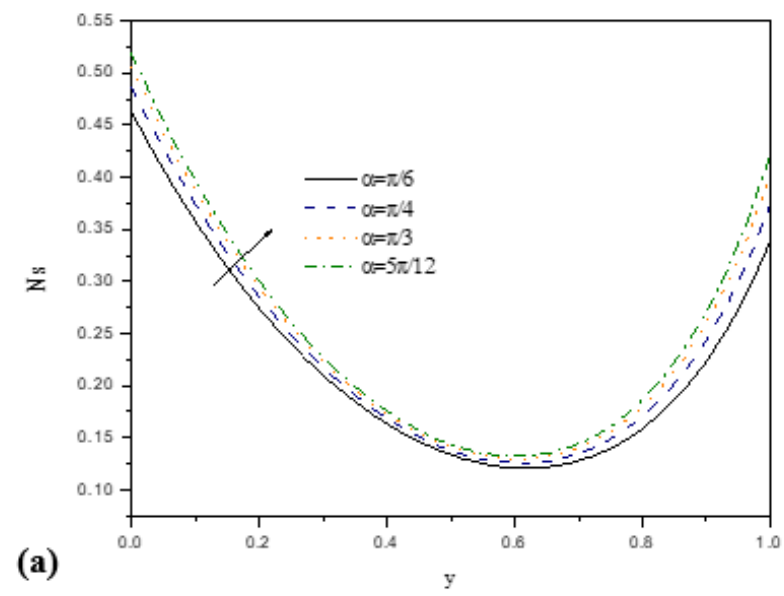

(b)

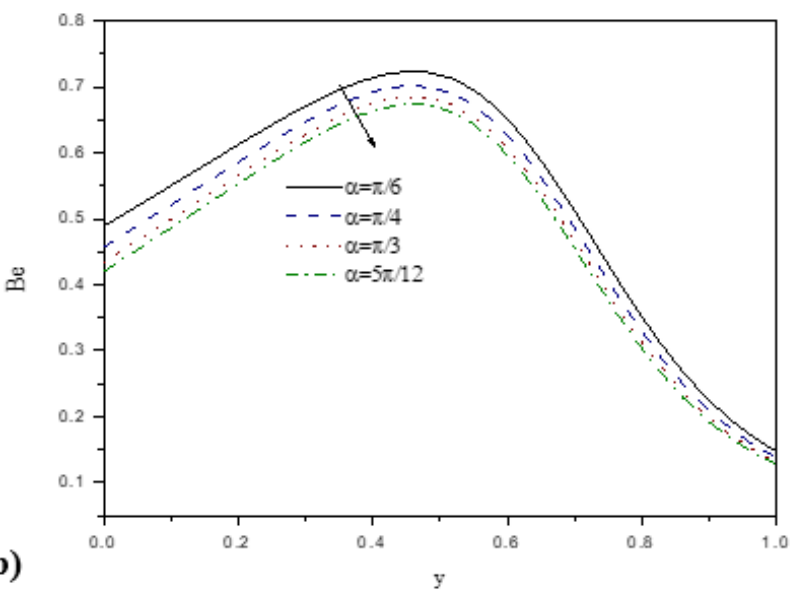

Fig 9. Impact of $\alpha$ on the on the entropy generation (a) and Bejan number (b) 


\section{Nomenclature}

a - distance between the plates $(m)$;

Bi - Biot number;

$\mathrm{Br}$ - Brinkman number

g - acceleration due to gravity $\left(\mathrm{m} / \mathrm{s}^{2}\right)$

Gr - Grashof number;

$\mathrm{K}$ - thermal conductivity;

$\mathrm{M}$ - Hartman number;

$\mathrm{P}$ - pressure $\left(\mathrm{kgm}^{-1} \mathrm{~s}^{-2}\right)$

$R e$ - Reynolds number;

$\mathrm{T}$ - temperature $(\mathrm{K})$;

$T_{h}$ - hot fluid temperature;

$T_{P}$ - characteristic temperature ratio;

$T_{a}$ - ambient temperature;

$\mathrm{u}$ - velocity $(\mathrm{m} / \mathrm{s})$;

We - Wessenberg number;

Greek symbols:

$\lambda$ - mixed convection parameter;

$\lambda_{C}$ - concentration buoyancy parameter;

$\theta$ - dimensionless temperature;

$\alpha$ - channel inclination;

$\varepsilon$ - variable thermal conductivity parameter;

$\mu$ - dynamic viscosity $\left(\mathrm{kg} \mathrm{m}^{-1} \mathrm{~s}^{-1}\right)$;

$\xi$ - chemical reaction parameter;

$\phi$ - concentration parameter;

$\psi$ - heat generation/absorption parameter;

$\sigma$ - permeability parameter;

\section{References}

1) Makinde OD, Eegunjobi AS. ENTROPY ANALYSIS OF THERMALLY RADIATING MAGNETOHYDRODYNAMIC SLIP FLOW OF CASSON FLUID IN A MICROCHANNEL FILLED WITH SATURATED POROUS MEDIA. Journal of Porous Media. 2016;19(9):799-810. Available from: https://dx.doi.org/10.1615/jpormedia.v19.i9.40.

2) Zaib A, Abelman S, Chamkha AJ, Rashidi MM. Entropy generation in a Williamson nanofluid near a stagnation point over a moving plate with binary chemical reaction and activation energy. Heat Transfer Research. 2018;49(12):1131-1149. Available from: https://dx.doi.org/10.1615/heattransres. 2018019743.

3) Khan MI, Qayyum S, Hayat T, Khan MI, Alsaedi A. Entropy optimization in flow of Williamson nanofluid in the presence of chemical reaction and Joule heating. International Journal of Heat and Mass Transfer. 2019;133:959-967. Available from: https://dx.doi.org/10.1016/j.ijheatmasstransfer.2018.12.168.

4) Kumar A, Tripathi R, Singh R, Chaurasiya VK. Simultaneous effects of nonlinear thermal radiation and Joule heating on the flow of Williamson nanofluid with entropy generation. Physica A: Statistical Mechanics and its Applications. 2020;551:123972. Available from: https://dx.doi.org/10.1016/j.physa.2019. 123972.

5) Gireesha BJ, Roja A. Second law analysis of MHD natural convection slip flow of Casson fluid through an inclined microchannel. Multidiscipline Modeling in Materials and Structures. 2020;16:1435-1455. Available from: https://dx.doi.org/10.1108/mmms-11-2019-0189.

6) Saima N, Dianchen L, Sadia W. Entropy generation in electromagnetohydrodynamic water based three nano fluids via porous asymmetric microchannel. European Journal of Mechanics- B/Fluids. 2020;85. Available from: https://doi.org/10.1016/j.euromechflu.2020.11.002.

7) Sahoo A, Nandkeolyar R. Entropy generation and dissipative heat transfer analysis of mixed convective hydromagnetic flow of a Casson nanofluid with thermal radiation and Hall current. Scientific Reports. 2021;11(1). Available from: https://dx.doi.org/10.1038/s41598-021-83124-0.

8) Salahuddin T, Malik MY, Hussain A, Awais M, Bilal S. Mixed Convection Boundary Layer Flow of Williamson Fluid with Slip Conditions Over a Stretching Cylinder by Using Keller Box Method. International Journal of Nonlinear Sciences and Numerical Simulation. 2017;18(1):9-17. Available from: https://dx.doi.org/10.1515/ijnsns-2015-0090.

9) Jha BK, Aina B. Role of suction/injection on steady fully developed mixed convection flow in a vertical parallel plate microchannel. Ain Shams Engineering Journal. 2018;9(4):747-755. Available from: https://dx.doi.org/10.1016/j.asej.2016.05.001.

10) Xu H, Sun Q. Generalized Hybrid Nanofluid Model with the Application of Fully Developed Mixed Convection Flow in a Vertical Microchannel. Communications in Theoretical Physics. 2019;71(8). Available from: https://dx.doi.org/10.1088/0253-6102/71/8/903.

11) Mallikarjun P, Murthy R, Mahabaleshwar U, Lorenzini G. Numerical Study of Mixed Convective Flow of a Couple Stress Fluid in a Vertical Channel with First Order Chemical Reaction and Heat Generation/Absorption. Mathematical Modelling of Engineering Problems. 2019;6(2):175-182. Available from: https://dx.doi.org/10.18280/mmep.060204. 
12) C SK, Biradar M, B PM. Mixed convective flow with variable viscosity and variable thermal conductivity in a channel in the presence of first order chemical reaction with heat generation or absorption. Journal of Applied Mathematics and Computational Mechanics. 2021;20(1):91-102. Available from: https://dx.doi.org/10.17512/jamcm.2021.1.09.

13) Ibrahim W, Anbessa T. Hall and ion-slip effects on mixed convection flow of Williamson nanofluid over a nonlinear porous stretching sheet with variable thermal conductivity. Heat Transfer. 2021;50(6):5627-5651. Available from: https://dx.doi.org/10.1002/htj.22141.

14) Cimpean DS, Pop I. Fully developed mixed convection flow of a nanofluid through an inclined channel filled with a porous medium. International Journal of Heat and Mass Transfer. 2012;55(4):907-914. Available from: https://dx.doi.org/10.1016/j.ijheatmasstransfer.2011.10.018.

15) Srinivasacharya D, Bindu KH. Entropy generation in a micropolar fluid flow through an inclined channel. Alexandria Engineering Journal. 2016;55(2):973982. Available from: https://dx.doi.org/10.1016/j.aej.2016.02.027. doi:10.1016/j.aej.2016.02.027.

16) Zaidi HN, Ahmad N. MHD convection fluid flow and heat transfer in an inclined microchannel with heat generation. American Journal of Applied Mathematics. 2017;5:124-131.

17) Idowu AS, Akolade MT, Oyekunle TL, Abubakar JU. Nonlinear convection flow of dissipative Casson nanofluid through an inclined annular microchannel with a porous medium. Heat Transfer. 2021;50(4):3388-3406. Available from: https://dx.doi.org/10.1002/htj.22033.

18) Gireesha BJ, Sindhu S, Sowmya G, Felicita A. Magnetohydrodynamic flow of Williamson fluid in a microchannel for both horizontal and inclined loci with wall shear properties. Heat Transfer. 2021;50(2):1428-1442. Available from: https://dx.doi.org/10.1002/htj.21937.

19) Ilias MR, Rawi NA, Shafie S. MHD Free Convection Flow and Heat Transfer of Ferrofluids over a Vertical Flat Plate with Aligned and Transverse Magnetic Field. Indian Journal of Science and Technology. 2016;9(36):1-7. Available from: https://dx.doi.org/10.17485/ijst/2016/v9i36/97347.

20) Venkateswarlu B, Narayana PVS, Devika B. Effects of Chemical Reaction and Heat Source on MHD Oscillatory Flow of a Viscoelastic Fluid in a Vertical Porous Channel. International Journal of Applied and Computational Mathematics. 2017;3(S1):937-952. Available from: https://dx.doi.org/10.1007/ s40819-017-0391-8.

21) Mallikarjun P, Murthy RV, Mahabaleshwar US, Chamkha AJ, Lorenzini G. Finite-Element Analysis of Fully Developed Mixed Convection through a Vertical Channel in the Presence of Heat Generation/Absorption with a First-Order Chemical Reaction. Defect and Diffusion Forum. 2018;388:394-406. Available from: https://dx.doi.org/10.4028/www.scientific.net/ddf.388.394.

22) Mo X, Hu X. Electroviscous effect on pressure driven flow and related heat transfer in microchannels with surface chemical reaction. International Journal of Heat and Mass Transfer. 2019;130:813-820. Available from: https://dx.doi.org/10.1016/j.ijheatmasstransfer.2018.10.113.

23) Abbas Z, Rafiq MY, Hasnain J, Umer H. Impacts of lorentz force and chemical reaction on peristaltic transport of Jeffrey fluid in a penetrable channel with injection/suction at walls. Alexandria Engineering Journal. 2021;60(1):1113-1122. Available from: https://dx.doi.org/10.1016/j.aej.2020.10.035.

24) Zigta B. Mixed Convection on MHD Flow with Thermal Radiation, Chemical Reaction and Viscous Dissipation Embedded in a Porous Medium. International Journal of Applied Mechanics and Engineering. 2020;25(1):219-235. Available from: https://dx.doi.org/10.2478/ijame-2020-0014.

25) Umavathi JC, Al-Mudhaf A, Chamkha AJ. Effect of variable thermal conductivity of water based nano fluids saturated with porous medium on natural convection heat transfer enhancement. Computational Thermal Sciences: An International Journal. 2021;13(2):21-43. Available from: https: //dx.doi.org/10.1615/computthermalscien.2020035975.

26) Alireza F. Entropy generation analysis of fully developed laminar forced convection in a confocal elliptical duct with uniform wall heat fluxes. Indian Journal of Science and Technology. 2011;4(12):1649-1653. Available from: https://doi.org/10.17485/ijst/2011/v4i12.22.

27) Makinde O, Eegunjobi A. Effects of Convective Heating on Entropy Generation Rate in a Channel with Permeable Walls. Entropy. 2013;15(1):220-233. Available from: https://dx.doi.org/10.3390/e15010220.

28) Dahiya A, Sehgal SS, Singh H. CFD and Heat Transfer Analysis of Microchannel Heat Sink with Different Inlet and Outlet Flow Arrangements. Indian Journal of Science and Technology. 2016;9(36):1-5. Available from: https://dx.doi.org/10.17485/ijst/2016/v9i36/101406.

29) Singh KK, Sinha MK. Analysis of Entropy Generation Minimization in Radial Heat Sink. Indian Journal of Science and Technology. 2016;9(38):1-7. Available from: https://dx.doi.org/10.17485/ijst/2016/v9i38/92487.

30) Renukadevi RL, Neeraja A, Swapna Y. Numerical study of MHD Casson liquid stream over an infinite vertical porous plate with Newtonian heating. Indian Journal of Science and Technology. 2020;13(2):149-160.

31) Abdelmalek Z, Khan SU, Waqas H, Riaz A, Khan IA, Tlili I. A mathematical model for bioconvection flow of Williamson nanofluid over a stretching cylinder featuring variable thermal conductivity, activation energy and second-order slip. Journal of Thermal Analysis and Calorimetry. 2021;144(1):205217. Available from: https://dx.doi.org/10.1007/s10973-020-09450-z.

32) Ellahi R, Alamri SZ, Basit A, Majeed A. Effects of MHD and slip on heat transfer boundary layer flow over a moving plate based on specific entropy generation. Journal of Taibah University for Science. 2018;12(4):476-482. Available from: https://dx.doi.org/10.1080/16583655.2018.1483795. doi:10.1080/16583655.2018.1483795.

33) Ellahi R, Sait SM, Shehzad N, Ayaz Z. A hybrid investigation on numerical and analytical solutions of electro-magnetohydrodynamics flow of nanofluid through porous media with entropy generation. International Journal of Numerical Methods for Heat \& Fluid Flow. 2019;30(2):834-854. Available from: https://dx.doi.org/10.1108/hff-06-2019-0506. doi:10.1108/hff-06-2019-0506.

34) Shaheen S, Maqbool K, Ellahi R, Sait SM. Heat transfer analysis of tangent hyperbolic nanofluid in a ciliated tube with entropy generation. Journal of Thermal Analysis and Calorimetry. 2021;144:2337-2346. Available from: https://dx.doi.org/10.1007/s10973-021-10681-x.

35) Bhatti MM, Riaz A, Zhang L, Sait SM, Ellahi R. Biologically inspired thermal transport on the rheology of Williamson hydromagnetic nanofluid flow with convection: an entropy analysis. Journal of Thermal Analysis and Calorimetry. 2021;144(6):2187-2202. Available from: https://dx.doi.org/10.1007/ s10973-020-09876-5. 